Research Article

\title{
Transverse SINS/DVL Integrated Polar Navigation Algorithm Based on Virtual Sphere Model
}

\author{
Ling Huang $\mathbb{D}^{\mathrm{D}}$, Xiang Xu, Heming Zhao $\mathbb{D}$, and Haoran Ge \\ School of Electronic and Information Engineering, Soochow University, Suzhou, China \\ Correspondence should be addressed to Heming Zhao; hmzhao@suda.edu.cn
}

Received 26 August 2020; Revised 14 September 2020; Accepted 29 September 2020; Published 2 November 2020

Academic Editor: Gaoge $\mathrm{Hu}$

Copyright () 2020 Ling Huang et al. This is an open access article distributed under the Creative Commons Attribution License, which permits unrestricted use, distribution, and reproduction in any medium, provided the original work is properly cited.

\begin{abstract}
Due to the principle error caused by the Earth's sphere model in transverse polar navigation, the ellipsoid model is usually used for transverse transformation. In order to avoid the complex transverse transformation of the ellipsoid model, a virtual sphere model, which can simplify the transformation of the ellipsoid model, is constructed in this paper. With the requirements of high accuracy and long-time navigation for AUV in the polar region, the integrated navigation scheme of SINS/DVL, which is based on the virtual sphere model, is proposed. The proposed method can improve the navigation accuracy and suppress the oscillation error. The error equations of transverse SINS are established based on the virtual sphere model. Then, the transverse SINS/DVL integrated navigation algorithm is derived according to the new error equations. The simulation results show that the navigation accuracy of the proposed method is equivalent with the traditional ellipsoid model method and is better than that of the traditional sphere model method. However, the complexity of the proposed method is simpler than the traditional ellipsoid model method. Moreover, it is verified that the navigation accuracy of SINS/DVL integrated navigation system based on the virtual sphere model meets the requirements of AUV.
\end{abstract}

\section{Introduction}

In recent years, both scientific research and seafaring application have been carried out in the polar region, which requires the safety and reliability, and navigation technology is an important information for polar region exploration. Due to the importance of the polar region, the navigation application for AUV has become a hot topic of research in recent years [1-3]. Therefore, ensuring the navigation performance whether in or going to the poles is of great strategic significance. According to the special geographical location and environment of the polar region, traditional SINS mechanization is not suitable for the polar region due to the rapid convergence of the Earth's meridians with the latitude increasing.

To solve the problem, transverse navigation method was proposed by MIT [4]. The sphere model is usually applied to the traditional transverse navigation method, which can bring advantages of briefness $[5,6]$. However, the shape of the Earth is approximated to an ellipsoid and the corresponding simplification inevitably brings principle errors and decreases the navigation accuracy. In $[7,8]$, the ellipsoid model method is proposed to reduce the principle error, which is caused by the sphere model. In [9], the influence of the Earth model for the inertial navigation system in the polar region is analysed based on the transverse frame. In [10], the radius of transverse meridian and the radius of transverse prime vertical are deduced in detail, and the navigation performance of the ellipsoid and sphere model is compared. In [11], the definitions of the transverse geocentric latitude and transverse geographical latitude are given, and the relationship between geocentric latitude and transverse geocentric latitude is established. Then, the relationship between traditional navigation parameters and transversal parameters is presented, and the mechanization based on the transverse geographic frame is deduced. 
Finally, the explicit expressions of transverse curvature radius are given. In $[12,13]$, the systematic errors of the transverse frame caused by each inertial measurement unit and the geometric error are derived. Obviously, the real shape of the Earth is an irregular sphere, so the geometric error of the sphere model approximation will produce the inertial navigation error. The ellipsoid model closer to the real shape of the Earth and the geometric error, which cannot be ignored in the sphere model, can be ignored, and then the principle error of the transverse navigation system caused by geometric errors can be reduced. From the above literatures, we can see that the traditional ellipsoid model can reduce the principle error, but the derivation and solution of curvature radius are too complicated and the navigation process is too cumbersome $[10,11]$. In [14], a virtual sphere model is proposed to simplify the transverse transformation, and velocity compensations can be applied to reduce the principle errors, and the accuracy is better than that of the sphere model. However, the error equations are not deduced, and the error equations of transverse navigation based on virtual sphere are deduced in this paper, which can refer to the literature [15]. Considering the application characteristics of AUV, DVL auxiliary system can be used to improve the navigation accuracy and eliminate periodic oscillation errors. At present, the traditional SINS/DVL integrated navigation scheme is widely used in AUV highprecision autonomous navigation in middle-low latitude regions [16]. In [17], the transverse integrated polar navigation of SINS/DVL based on the traditional sphere model is proposed.

In this paper, instead of solving the complex transversal radius of the ellipsoid model, a virtual sphere model is proposed, which uses the radius of curvature in prime vertical as the radius to construct a virtual sphere and uses the expansion coefficient to compensate the velocities of the carrier. According to the basic equation of virtual sphere model, the corresponding transverse error equation is derived, and then the Kalman filter model of transverse SINS/ DVL integrated navigation system based on the virtual sphere model is deduced in detail in this paper. The simulation results show that the proposed virtual sphere model can suppress the oscillation error and reduce the velocity constant error. Moreover, the accuracy of the proposed transverse SINS/DVL integrated navigation method based on the virtual sphere model is better than that of the traditional sphere model method and is similar to that of the ellipsoid model method, which can meet the needs of polar navigation. This article is divided into the following sections: Section 2 introduces the derivation of the proposed visual sphere model. In Section 3, the transverse error equation of AUV based on the visual sphere model is deduced. The proposed method of transverse SINS/DVL integrated polar navigation based on the visual sphere model is established in Section 4. The simulation of the proposed method is designed in Section 5. Section 6 presents a summary of our results.

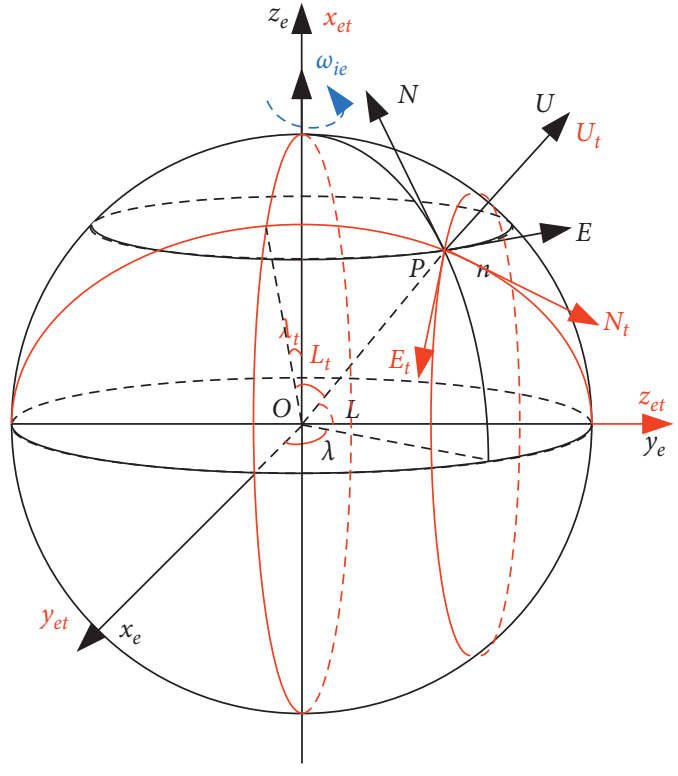

Figure 1: Transversal coordinate system.

\section{Polar Transverse Navigation System Based on Virtual Sphere Model}

2.1. Transverse Navigation Coordinate System. As shown in Figure 1, two points in the traditional equatorial plane were selected as the transversal north-south poles, and the intersection point of $90^{\circ}$ east meridian and the equatorial plane was defined as the transversal north pole point, and then the meridian coil composed of $0^{\circ}$ meridian and $180^{\circ}$ meridian was defined as the transversal equatorial plane. According to the new poles, the transverse Earth frame, the transverse geographic frame, and the transverse latitude and transverse longitude can be redefined.

The transverse coordinate system is defined as $t$, and the origin of the transverse frame is $O$. According to Figure 1, the relationship between the Earth frame and the transverse Earth frame is shown as

$$
C_{e}^{e_{t}}=\left[\begin{array}{lll}
0 & 0 & 1 \\
1 & 0 & 0 \\
0 & 1 & 0
\end{array}\right]
$$

The transverse navigation frame is defined as $n_{t}$ and the traditional navigation frame is defined as $n$. The relationship between the traditional navigation frame $n$ and the transverse navigation frame $n_{t}$ is shown as

$$
C_{n}^{n_{t}}=\left[\begin{array}{ccc}
\cos p & -\sin p & 0 \\
\sin p & \cos p & 0 \\
0 & 0 & 1
\end{array}\right],
$$

where $p$ represents the angle between $n_{t}$ and $n$, which can be obtained by 


$$
\begin{aligned}
& \cos p=\frac{-\sin L_{t} \cos \lambda_{t}}{\sqrt{1-\cos ^{2} L_{t} \cos ^{2} \lambda_{t}}}, \\
& \sin p=\frac{\sin \lambda_{t}}{\sqrt{1-\cos ^{2} L_{t} \cos ^{2} \lambda_{t}}} .
\end{aligned}
$$

Transverse latitude $L_{t}$ is the angle between the transverse equatorial plane and the line between the point $P$ and the geocentric; transverse longitude $\lambda_{t}$ is the angle between the transverse primary meridian plane and the transverse meridian plane passing through the point $\mathrm{P}$. According to the transverse coordinate system, the relationship between the longitude and latitude of the transverse navigation system and that of the traditional navigation system is described as follows:

$$
\left\{\begin{array}{l}
\sin L=\cos L_{t} \cos \lambda_{t} \\
\tan \lambda=\tan L_{t} \csc \lambda_{t} .
\end{array}\right.
$$

2.2. Transverse Navigation Coordinate System Based on Virtual Sphere Model. According to the transverse coordinate system, the relationship between the virtual sphere and the section of the rotating ellipsoid, which passes through the point $\mathrm{P}$ and the axis of symmetry, is shown in Figure 2. In Figure 2, the red dotted line represents the virtual sphere, $L_{e}$ represents the geocentric latitude of ellipsoid, and $L$ represents the geographic latitude of ellipsoid and the geocentric latitude of virtual sphere.

According to Figure 2, the elliptical flattening $f$ can be expressed as

$$
f=\frac{R_{e}-R_{p}}{R_{e}}
$$

where $R_{e}$ and $R_{p}$ represent the semimajor axis and the semiminor axis of the Earth ellipsoid model, respectively.

At the point $\mathrm{P}$, the Earth frame is expressed as

$$
\left\{\begin{array}{l}
x=R_{e} \cos \theta \\
z=R_{p} \sin \theta=R_{e}(1-f) \sin \theta .
\end{array}\right.
$$

According to the geometrical relation between the ellipsoid and virtual sphere, we can get

$$
\begin{aligned}
& \tan L=\frac{1}{(\mathrm{~d} z / \mathrm{d} x)}=\frac{(\mathrm{d} x / \mathrm{d} \theta)}{(\mathrm{d} z / \mathrm{d} \theta)}=\frac{1}{1-f} \tan \theta \\
& \tan L_{e}=\frac{z}{x}=\frac{R_{p}}{R_{e}} \tan \theta=(1-f) \tan \theta .
\end{aligned}
$$

(7) and (8) are sorted out as follows:

$$
\tan L=\frac{1}{(1-f)^{2}} \tan L_{e} .
$$

Let $O P=R_{t}, O_{e} P=R_{\mathrm{et}}$, which gives

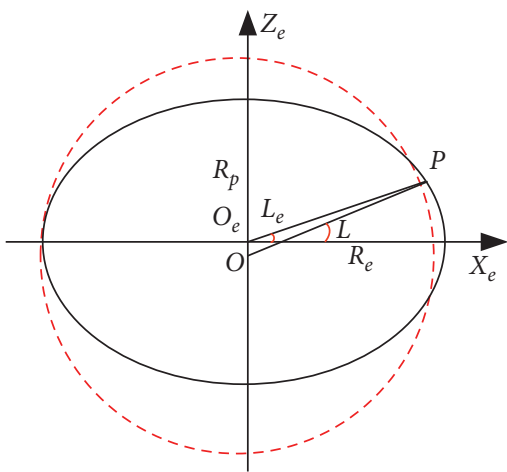

FIgURE 2: Schematic diagram of the virtual sphere.

$$
R_{t}=\frac{R_{\mathrm{et}} \cos L_{e}}{\cos L}
$$

According to the ellipsoid model equation, we obtain

$$
\frac{\left(R_{\mathrm{et}} \cos L_{e}\right)^{2}}{R_{e}^{2}}+\frac{\left(R_{\mathrm{et}} \sin L_{e}\right)^{2}}{R_{p}^{2}}=1 .
$$

By combining equations (9)-(11) and (4), the relationship between the radius of the virtual sphere model and transverse longitude and latitude can be obtained as follows:

$$
R_{t}=\frac{R_{e}}{\sqrt{1-\left(2 f-f^{2}\right)\left(\cos L_{t} \cos \lambda_{t}\right)^{2}}} .
$$

In other words, a virtual sphere is constructed by taking the radius of curvature in prime vertical as the radius at the carrier position. When the definition of Earth's meridian circle radius $R_{M}$ is considered, the virtual sphere can be regarded as anartificial sphere through the point $\mathrm{P}$ with a radius of the prime vertical circle. The expansion coefficient relative to the radius of the meridian circle can be defined by the following equation:

$$
k=\frac{R_{t}+h}{R_{M}+h} \text {. }
$$

In the traditional navigation system, the east velocity is tangent to the latitude circle, which is circular, so there is no need for virtual sphere mapping. While the north velocity is along the longitude tangent direction, the virtual sphere mapping is needed to reduce the principle error of the traditional sphere model by the expansion coefficient.

Thus, the velocity compensation in the transverse coordinate system is expressed as

$$
\mathbf{V}^{n_{t \prime}}=\mathbf{C}_{n}^{n_{t}} \cdot K \cdot \mathbf{C}_{n_{t}}^{n} \cdot \mathbf{V}^{n_{t}},
$$

where $\mathbf{V}^{n_{t}}$ and $\mathbf{V}^{n_{t \prime}}$ are the transverse velocities before and after compensation, respectively. The attitude matrix $\mathbf{C}_{n}^{n_{t}}$ is the direction cosine matrix from $n$ frame to $n_{t}$ frame, and the expansion coefficient matrix is expressed as follows:

$$
\mathbf{K}=\left[\begin{array}{lll}
1 & 0 & 0 \\
0 & k & 0 \\
0 & 0 & 1
\end{array}\right] \text {. }
$$


The important step of polar inertial navigation based on the virtual sphere model is to calculate the radius $R_{t}$ of the virtual sphere model. The calculation of $R_{t}$ needs the transverse longitude and latitude value of the last moment, and the velocity and position are updated by using the compensation velocity.

The mechanization of the transverse coordinate system based on the virtual sphere can be determined by the attitude, velocity, and position update equations, respectively (the specific derivation process is omitted).

$$
\left\{\begin{array}{l}
\dot{\mathbf{C}}_{b}^{n_{t}}=\mathbf{C}_{b}^{n_{t}}\left[\boldsymbol{\omega}_{n_{t} b}^{b} \times\right], \\
\dot{\mathbf{V}}^{n_{t}}=\mathbf{C}_{b}^{n_{t}} \mathbf{f}^{b}-\left(\boldsymbol{\omega}_{e_{t} n_{t}}^{n_{t}}+2 \boldsymbol{\omega}_{i e_{t}}^{n_{t}}\right) \times \mathbf{V}^{n_{t}}+\mathbf{g}^{n_{t}}, \\
L_{t}=\frac{\mathbf{V}_{n}^{n_{t}^{\prime}}}{\left(R_{t}+h\right)}, \\
\lambda_{t}=\frac{\mathbf{V}_{e}^{n_{t}^{\prime}} \sec L_{t}}{\left(R_{t}+h\right)},
\end{array}\right.
$$

where $\left[\omega_{n_{t} b}^{b} \times\right]$ is the antisymmetric matrix of $\omega_{n_{t} b}^{b}, \omega_{e_{t} n_{t}}^{n_{t}}=$ $\left[-\left(V_{n}^{n_{t}^{\prime}} / R_{t}+h\right)\left(V_{e}^{n_{t}^{\prime}} / R_{t}+h\right)\left(V_{e}^{n_{t}^{\prime}} / R_{t}+h\right) \tan L_{t}\right]^{T}$ is the angular rate of the transverse navigation relative transverse Earth frame, $\quad \omega_{i e_{t}}^{n_{t}}=\mathbf{C}_{e_{t}}^{n_{t}} \mathbf{C}_{e}^{e_{t}} \omega_{i e}^{e}=\left[-\omega_{i e}\right.$ $\left.\sin \lambda_{\bar{t}} \omega_{i e} \sin L_{t} \cos \lambda_{t} \omega_{i e} \cos L_{t} \cos \lambda_{t}\right]^{T}$ is the projection of the Earth's rotation angular rate in transverse navigation, $g^{n_{t}}=\left[\begin{array}{lll}0 & 0 & -g\end{array}\right]^{T}$ is the gravity vector in the transverse coordinate system, $\boldsymbol{\omega}_{n_{t} b}^{b}=\boldsymbol{\omega}_{i b}^{b}-\mathbf{C}_{n_{t}}^{b} \boldsymbol{\omega}_{i n_{t}}^{n_{t}}=\boldsymbol{\omega}_{i b}^{b}-\mathbf{C}_{n_{t}}^{b}\left(\boldsymbol{\omega}_{i e_{t}}^{n_{t}}+\right.$ $\left.\boldsymbol{\omega}_{e_{t} n_{t}}^{n_{t}}\right)$ is the attitude angular rate of the b-frame relative to transverse navigation frame, $\omega_{i b}^{b}$ is the gyro angular rate. The relationship between the transverse navigation frame and the transverse Earth frame is given by

$$
C_{e_{t}}^{n_{t}}=\left[\begin{array}{ccc}
-\sin \lambda_{t} & \cos \lambda_{t} & 0 \\
-\sin L_{t} \cos \lambda_{t} & -\sin L_{t} \sin \lambda_{t} & \cos L_{t} \\
\cos L_{t} \cos \lambda_{t} & \cos L_{t} \sin \lambda_{t} & \sin L_{t}
\end{array}\right] .
$$

\section{Error Equations of AUV Transverse Navigation Algorithm Based on the Virtual Sphere Model}

3.1. Attitude Error Equations. In the practical calculation, there exist errors in the process of attitude updating, and the updating equation in actual calculation is described as

$$
\begin{aligned}
& \dot{\hat{\mathbf{C}}}_{b}^{n_{t}}=\widehat{\mathbf{C}}_{b}^{n_{t}}\left[\widehat{\omega}_{n_{t} b}^{b} \times\right], \\
& \widehat{\mathbf{C}}_{b}^{n_{t}}=\mathbf{C}_{n_{t}}^{n_{t}^{\prime}} \mathbf{C}_{b}^{n_{t}}=\left(\mathbf{I}-\phi^{n_{t}} \times\right) \mathbf{C}_{b}^{n_{t}}, \\
& \widehat{\boldsymbol{\omega}}_{n_{t} b}^{b}=\widehat{\boldsymbol{\omega}}_{i b}^{b}-\mathbf{C}_{n_{t}}^{b} \widehat{\omega}_{i n_{t}}^{n_{t}}, \\
& \widehat{\boldsymbol{\omega}}_{i b}^{b}=\boldsymbol{\omega}_{i b}^{b}+\delta \boldsymbol{\omega}_{i b}^{b}=\boldsymbol{\omega}_{i b}^{b}+\boldsymbol{\varepsilon}^{b},
\end{aligned}
$$

$$
\begin{aligned}
& \widehat{\boldsymbol{\omega}}_{i n_{t}}^{n_{t}}=\widehat{\boldsymbol{\omega}}_{i e_{t}}^{n_{t}}+\widehat{\boldsymbol{\omega}}_{e_{t} n_{t}}^{n_{t}}, \\
& \widehat{\boldsymbol{\omega}}_{i e_{t}}^{n_{t}}=\boldsymbol{\omega}_{i e_{t}}^{n_{t}}+\delta \boldsymbol{\omega}_{i e_{t}}^{n_{t}}, \\
& \widehat{\boldsymbol{\omega}}_{e_{t} n_{t}}^{n_{t}}=\boldsymbol{\omega}_{e_{t} n_{t}}^{n_{t}}+\delta \boldsymbol{\omega}_{e_{t} n_{t}}^{n_{t}},
\end{aligned}
$$

where $\phi^{n_{t}} \times$ is the antisymmetric matrix of $\phi^{n_{t}}, \boldsymbol{\omega}_{i b}^{b}, \boldsymbol{\omega}_{i e_{t}}^{n_{t}}$ and $\boldsymbol{\omega}_{e_{t} n_{t}}^{n_{t}}$ are the errors of $\boldsymbol{\omega}_{i b}^{b}, \boldsymbol{\omega}_{i e_{t}}^{n_{t}}$, and $\boldsymbol{\omega}_{e_{t} n_{t}}^{n_{t}}$, respectively. $\varepsilon^{b}$ is the gyro drifts. The velocity and the position errors of AUV in transverse SINS can be described as $\delta \mathbf{V}^{n_{t}}=\left[\begin{array}{llll}\delta V_{e}^{n_{t}} & \delta V_{n}^{n_{t}} & \delta V_{u}^{n_{t}}\end{array}\right]^{T}$ and $\quad \delta \mathbf{P}^{n_{t}}=\left[\begin{array}{lll}\delta L_{t} & \delta \lambda_{t} & \delta h_{t}\end{array}\right]^{T}$, respectively. Thus, $\delta \boldsymbol{\omega}_{i e_{t}}^{n_{t}}$ and $\delta \boldsymbol{\omega}_{e_{t} n_{t}}^{n_{t}}$ are expressed as

$$
\begin{aligned}
& \delta \boldsymbol{\omega}_{i e_{t}}^{n_{t}}=\widehat{\boldsymbol{\omega}}_{i e_{t}}^{n_{t}}-\boldsymbol{\omega}_{i e_{t}}^{n_{t}}=\mathbf{M}_{1} \delta \mathbf{P}^{n t}, \\
& \delta \boldsymbol{\omega}_{e_{t} n_{t}}^{n_{t}}=\widehat{\boldsymbol{\omega}}_{e_{t} n_{t}}^{n_{t}}-\boldsymbol{\omega}_{e_{t} n_{t}}^{n_{t}}=\mathbf{M}_{a v} \delta \mathbf{v}^{n_{t}}+\mathbf{M}_{2} \delta \mathbf{P}^{n_{t}},
\end{aligned}
$$

where

$M_{a v}=\left[\begin{array}{ccc}0 & -\frac{1}{R_{t}+h} & 0 \\ \frac{1}{R_{t}+h} & 0 & 0 \\ \frac{\tan L_{t}}{R_{t}+h} & 0 & 0\end{array}\right]$,

$\mathbf{M}_{1}=\left[\begin{array}{ccc}0 & -\omega_{i e} \cos \lambda_{t} & 0 \\ -\omega_{i e} \cos L_{t} \cos \lambda_{t} & \omega_{i e} \sin L_{t} \sin \lambda_{t} & 0 \\ -\omega_{i e} \sin L_{t} \cos \lambda_{t} & -\omega_{i e} \cos L_{t} \sin \lambda_{t} & 0\end{array}\right]$,

$$
\mathbf{M}_{2}=\left[\begin{array}{ccc}
0 & 0 & \frac{v_{n}^{n_{t}^{\prime}}}{\left(R_{t}+h\right)^{2}} \\
0 & 0 & -\frac{v_{e}^{n_{t}^{\prime}}}{\left(R_{t}+h\right)^{2}} \\
\frac{v_{e}^{n_{t}^{\prime}}}{R_{t}+h} \sec ^{2} L_{t} & 0 & -\frac{v_{e}^{n_{t}^{\prime}} \tan L_{t}}{\left(R_{t}+h\right)^{2}}
\end{array}\right] .
$$

The error of attitude matrix and its derivative of AUV can be described as

$$
\begin{gathered}
\delta \mathbf{C}_{b}^{n_{t}}=\widehat{\mathbf{C}}_{b}^{n_{t}}-\mathbf{C}_{b}^{n_{t}}=-\phi^{n_{t}} \times \mathbf{C}_{b}^{n_{t}}, \\
\delta \dot{\mathbf{C}}_{b}^{n_{t}}=-\phi^{n_{t}} \times \dot{\mathbf{C}}_{b}^{n_{t}}-\dot{\phi}^{n_{t}} \times \mathbf{C}_{b}^{n_{t}} .
\end{gathered}
$$

By combining with equations (18)-(31), the attitude error equation of AUV navigation in the transverse SINS based on the virtual sphere model can be expressed in the form of vector as 


$$
\dot{\phi}^{n_{t}}=-\left(\boldsymbol{\omega}_{i n_{t}}^{n_{t}} \times\right) \phi^{n_{t}}+\left(\mathbf{M}_{1}+\mathbf{M}_{2}\right) \delta \mathbf{P}^{n_{t}}+\mathbf{M}_{a v} \delta \mathbf{V}^{n_{t}}-\mathbf{C}_{b}^{n_{t}} \boldsymbol{\varepsilon}^{b}
$$

3.2. Velocity Error Equation. Due to the errors from the IMU, the velocity error equation of AUV in practical application can be expressed as

$$
\begin{aligned}
& \dot{\hat{\mathbf{V}}}^{n_{t}}=\widehat{C}_{b}^{n_{t}} \widehat{f}^{b}-\left(\widehat{\omega}_{e_{t} n_{t}}^{n_{t}}+2 \widehat{\omega}_{i e_{t}}^{n_{t}}\right) \times \widehat{\mathbf{V}}^{n_{t}}+\widehat{\mathbf{g}}^{n_{t}}, \\
& \widehat{\mathbf{V}}^{n_{t}}=\mathbf{V}^{n_{t}}+\delta \mathbf{V}^{n_{t}}, \\
& \nabla^{b}=\widehat{\mathbf{f}}^{b}-\mathbf{f}^{b}, \\
& \widehat{\mathbf{g}}^{n_{t}}=\mathbf{g}^{n_{t}}+\delta \mathbf{g}^{n_{t}},
\end{aligned}
$$

where $\delta \mathbf{V}^{n_{t}}, \nabla^{b}$, and $\delta \mathbf{g}^{n_{t}}$ are the errors $\mathbf{V}^{n_{t}}, \mathbf{f}^{b}$ and $\mathbf{g}^{n_{t}}$, respectively. By combining with equations (30)-(36), (18), and (23), the velocity error equation in the transverse coordinate system based on the virtual sphere model can be described in the form of vector as

$$
\begin{aligned}
\delta \dot{\mathbf{V}}^{n_{t}}= & \mathbf{f}^{n_{t}} \times \phi^{n_{t}}+\left[\mathbf{V}^{n_{t}} \times\left(2 \mathbf{M}_{1}+\mathbf{M}_{2}\right)\right] \delta \mathbf{P}^{n_{t}} \\
& +\left[\mathbf{V}^{n_{t}} \times \mathbf{M}_{a v}-\left(2 \boldsymbol{\omega}_{i e_{t}}^{n_{t}}+\boldsymbol{\omega}_{e_{t} n_{t}}^{n_{t}}\right) \times\right] \cdot \delta \mathbf{V}^{n_{t}}+\mathbf{C}_{b}^{n_{t}} \nabla^{b},
\end{aligned}
$$

3.3. Position Error Equation. The position error equation of AUV navigation based on the virtual sphere model can be expressed in the form of vector as

$$
\delta \dot{\mathbf{P}}^{n_{t}}=\mathbf{M}_{p p} \delta \mathbf{P}^{n_{t}}+\mathbf{M}_{p v} \delta \mathbf{V}^{n_{t \prime}}
$$

where

$$
\begin{aligned}
\mathbf{M}_{p p} & =\left[\begin{array}{ccc}
0 & 0 & -\frac{v_{n}^{n_{t}^{\prime}}}{\left(R_{t}+h\right)^{2}} \\
\frac{v_{e}^{n_{t^{\prime}}} \tan L_{t} \sec L_{t}}{R_{t}+h} & 0 & -\frac{v_{e}^{n_{t}^{\prime}} \sec L_{t}}{\left(R_{t}+h\right)^{2}} \\
0 & 0 & 0
\end{array}\right], \\
\mathbf{M}_{p v} & =\left[\begin{array}{ccc}
0 & \frac{1}{R_{t}+h} & 0 \\
\frac{\sec L_{t}}{R_{t}+h} & 0 & 0 \\
0 & 0 & 1
\end{array}\right] .
\end{aligned}
$$

\section{Polar Integrated Navigation Based on Virtual Sphere Model}

At present, the research on high latitude navigation mainly focuses on the transverse inertial navigation under the traditional sphere model and ellipsoid model. The performance of SINS/DVL integrated navigation based on the virtual sphere model has not been verified by experiments. Due to the application characteristics of AUV, it is necessary to verify the effectiveness of the proposed SINS/DVL integrated navigation method based on the virtual sphere model in the polar region.

4.1. The State Equation. The state equation of AUV based on the virtual sphere model can be expressed as follows:

$$
\dot{\mathbf{X}}=\mathbf{F x}+\mathbf{W},
$$

where $\mathbf{X}$ is the state vector and $\mathbf{F}$ and $\mathbf{W}$ are the system matrix and system noise matrix. The state vector $\mathbf{X}$ includes the states that are attitude errors $\phi^{n_{t}}$, velocity errors $\delta \mathbf{V}^{n_{t}}$, position error $\delta \mathbf{P}^{n_{t}}$, gyro drifts $\varepsilon^{b}$, and accelerometer bias $\nabla^{b}$, which can be obtained as follows:

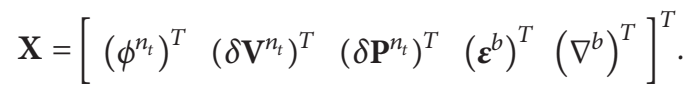

The system error equation can be established based on the attitude error, velocity error, and position error equations of AUV, and the state model can be described as follows:

$$
\left\{\begin{array}{l}
\dot{\phi}^{n_{t}}=-\left(\boldsymbol{\omega}_{i n_{t}}^{n_{t}} \times\right) \phi^{n_{t}}+\left(\mathbf{M}_{1}+\mathbf{M}_{2}\right) \delta \mathbf{P}^{n_{t}}+\mathbf{M}_{a v} \delta \mathbf{V}^{n_{t}}-\mathbf{C}_{b}^{n_{t}} \boldsymbol{\varepsilon}^{b}, \\
\delta \dot{\mathbf{V}}^{n_{t}}=\mathbf{f}^{n_{t}} \times \phi^{n_{t}}+\left[\mathbf{V}^{n_{t}} \times\left(2 \mathbf{M}_{1}+\mathbf{M}_{2}\right)\right] \delta \mathbf{P}^{n_{t}}+\left[\mathbf{V}^{n_{t}} \times \mathbf{M}_{a v}-\left(2 \boldsymbol{\omega}_{i e_{t}}^{n_{t}}+\boldsymbol{\omega}_{e_{t} n_{t}}^{n_{t}}\right) \times\right] \cdot \delta \mathbf{V}^{n_{t}}+\mathbf{C}_{b}^{n_{t}} \nabla^{b}, \\
\delta \dot{P}^{n_{t}}=\mathbf{M}_{p p} \delta \mathbf{P}^{n_{t}}+\mathbf{M}_{p v} \delta \mathbf{V}^{n_{t}^{\prime}} \\
\dot{\varepsilon}^{b}=0 \\
\dot{\nabla}=0 .
\end{array}\right.
$$


4.2. The Measurement Equation. Considering the characteristics of AUV, DVL is one of the common auxiliary systems to provide velocity, and the velocity error is chosen as the observation of the AUV transverse navigation system based on the virtual sphere model. The measurement error of DVL is $\delta \mathbf{v}_{\mathrm{DVL}}^{b}$, and the measured output is the three-dimensional velocities of the body frame system as follows:

$$
\widehat{\mathbf{v}}_{\mathrm{DVL}}^{b}=\mathbf{v}_{\mathrm{DVL}}^{b}+\delta \mathbf{v}_{\mathrm{DVL}}^{b},
$$

where $\mathbf{v}_{\mathrm{DVL}}^{b}$ is the actual velocity of the AUV under the body frame.

The output attitude angle of SINS is used to transform the velocity from the transverse navigation frame to the body frame; it gives

$$
\widehat{v}_{\text {SINS }}^{b}=\widehat{\mathbf{C}}_{n_{t}}^{\mathbf{b}} \widehat{\mathbf{v}}_{\text {SINS }}^{\mathbf{n}_{t}}=\mathbf{C}_{n_{t}}^{b} \mathbf{C}_{n_{t}^{\prime}}{ }^{n_{t}} \widehat{v}_{\text {SINS }}^{n_{t}}=\mathbf{C}_{n_{t}}^{b}\left(\mathbf{I}+\phi^{n_{t}} \times\right)\left(\mathbf{v}_{\text {SINS }}^{n_{t}}+\delta \mathbf{v}_{\text {SINS }}^{n_{t}}\right),
$$

where $\mathbf{I}$ is the identity matrix and $\delta \mathbf{v}_{\text {SINS }}^{n_{t}}$ is the error of $\mathbf{v}_{\text {SINS }}^{n_{t}}$ in the transverse navigation frame.

Taking the three-dimensional velocities of the body frame, which transformed from the output velocity of SINS in the transverse navigation frame, minus the threedimensional velocities of DVL as the measurement equation, and the second-order small quantity is ignored; it gives

$$
\mathbf{Z}=\widehat{\mathbf{v}}_{\text {sin } s}^{b}-\widehat{\mathbf{v}}_{\mathrm{DVL}}^{b}=-\mathbf{C}_{n_{t}}^{b} \mathbf{v}_{\text {SINS }}^{n_{t}} \times \phi^{n_{t}}+\mathbf{C}_{n_{t}}^{b} \delta \mathbf{v}_{\text {SINS }}^{n_{t}}-\delta \mathbf{v}_{\mathrm{DVL}}^{n_{t}} .
$$

The measurement equation is written to matrix form:

$$
\mathbf{Z}=\mathbf{H X}+\mathbf{V}
$$

where $\mathbf{V}$ is the measurement noise vector and $\mathbf{H}$ is the observation matrix:

$$
\mathbf{H}=\left[\begin{array}{ccccccc}
0 & v_{u}^{b} & -v_{n}^{b} & & & & \\
-v_{u}^{b} & 0 & v_{e}^{b} & \mathbf{C}_{n_{t}}^{b} & 0_{3 \times 3} & 0_{3 \times 3} & 0_{3 \times 3} \\
v_{n}^{b} & -v_{e}^{b} & 0 & & & &
\end{array}\right]
$$

\section{Simulation Results and Analysis}

When considering the limitation of polar experimental conditions, the simulation experiments are carried out to verify the performance of the proposed method.

\subsection{Simulation of Transverse Inertial Navigation Based on} Virtual Sphere Model. To verify the effectiveness of the proposed virtual sphere model in transverse SINS, the traditional sphere model and the traditional ellipsoid model are chosen as the comparative models. Simulations are carried out in the following environment: the simulation time is $24 \mathrm{~h}$. The sampling interval is $1 \mathrm{~s}$. The initial position of AUV is $\left(85^{\circ} \mathrm{N}, 118^{\circ} \mathrm{E}, 0 \mathrm{~m}\right)$ and the north velocity is $6 \mathrm{~m} / \mathrm{s}$. Except for different Earth models, other conditions are set to the same. In order to reflect the performance difference between the virtual sphere model and other traditional

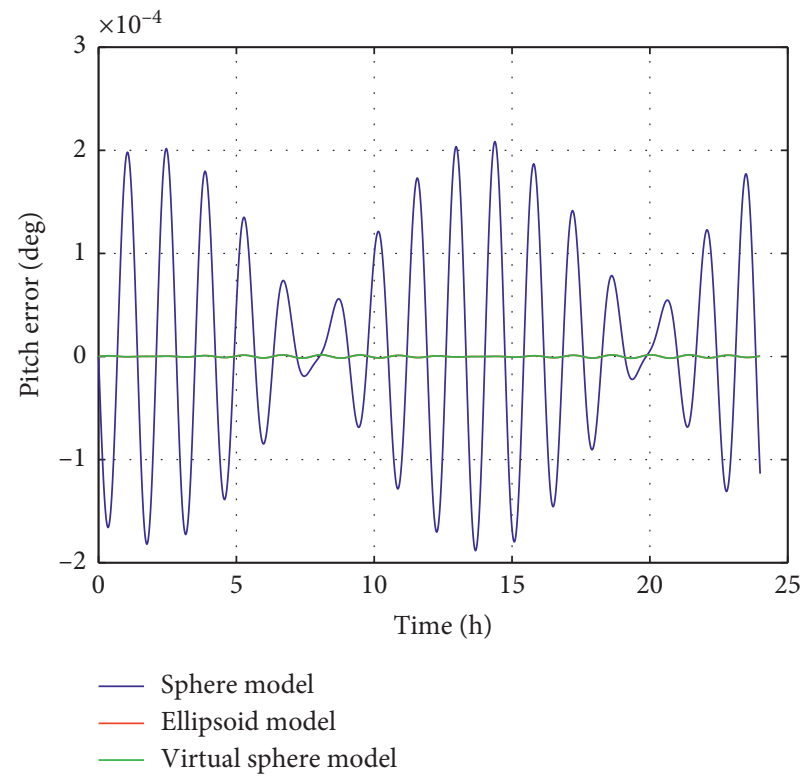

FIgURE 3: Pitch error.

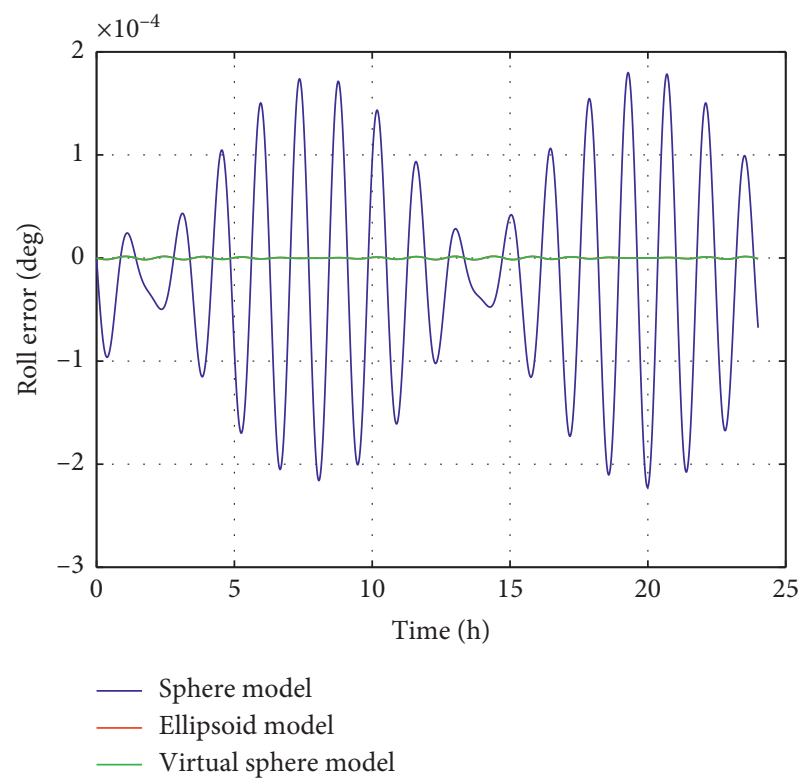

FIgURE 4: Roll error.

models, the simulation is realized without inertial sensor errors. The simulation results are shown in Figures 3-9.

The simulation results show that from Figures 3 to 9, without considering the error of inertial sensor, the traditional transverse navigation using the sphere model has obvious principle error. The proposed virtual sphere model in this paper can not only reduce the principle error but also reduce the constant errors of the east and north velocities and also has obvious effect on suppressing the oscillation errors. The error curves of the virtual sphere model and the traditional ellipsoid model almost coincide with each other and have almost the same accuracy in attitude, velocity, and position, and both of them are better than the traditional sphere model 


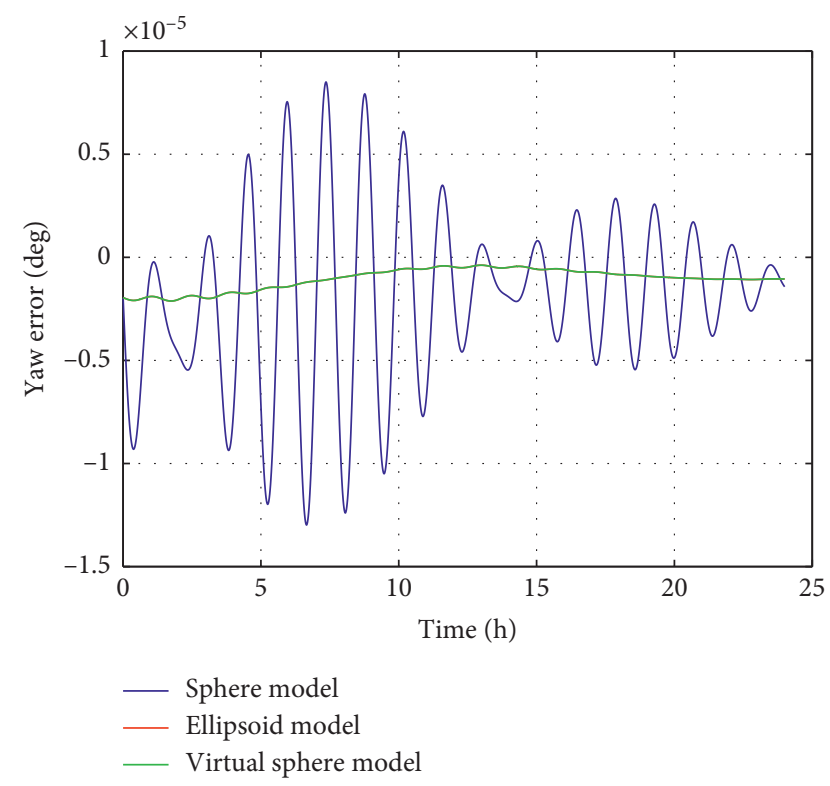

FIgURE 5: Yaw error.

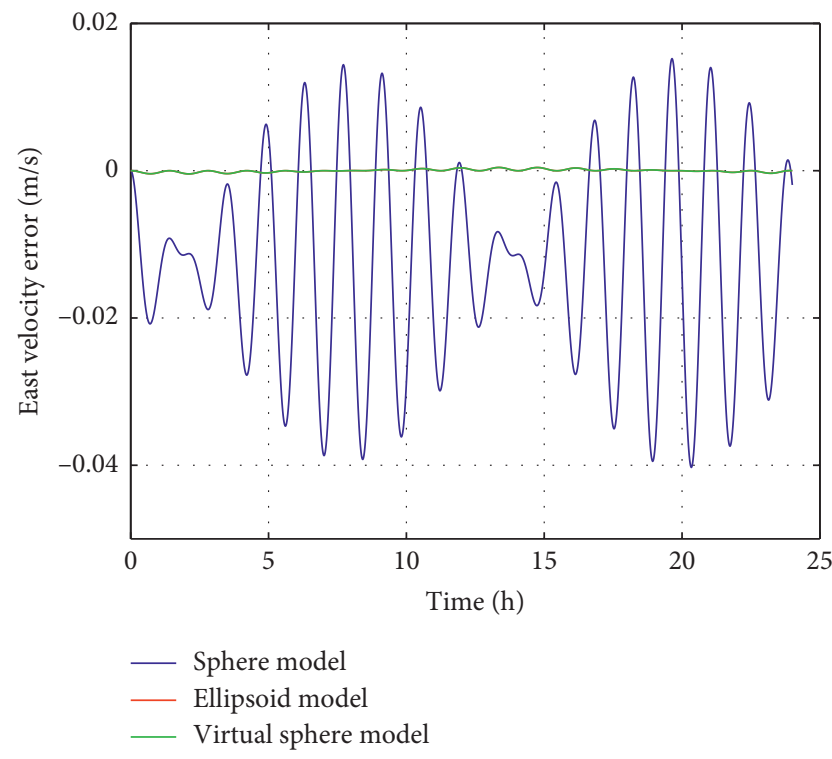

Figure 6: East velocity error.

in transverse navigation. It can be seen from Figure 10 that the time consumption of the proposed model is less than the traditional ellipsoid model, but the accuracy is similar.

5.2. The Integrated Navigation Algorithms. There are also three Earth models discussed in this part: the traditional sphere model, the traditional ellipsoid model, and the virtual sphere model. All the three models can be used in the transverse SINS/DVL integrated navigation to suppress the

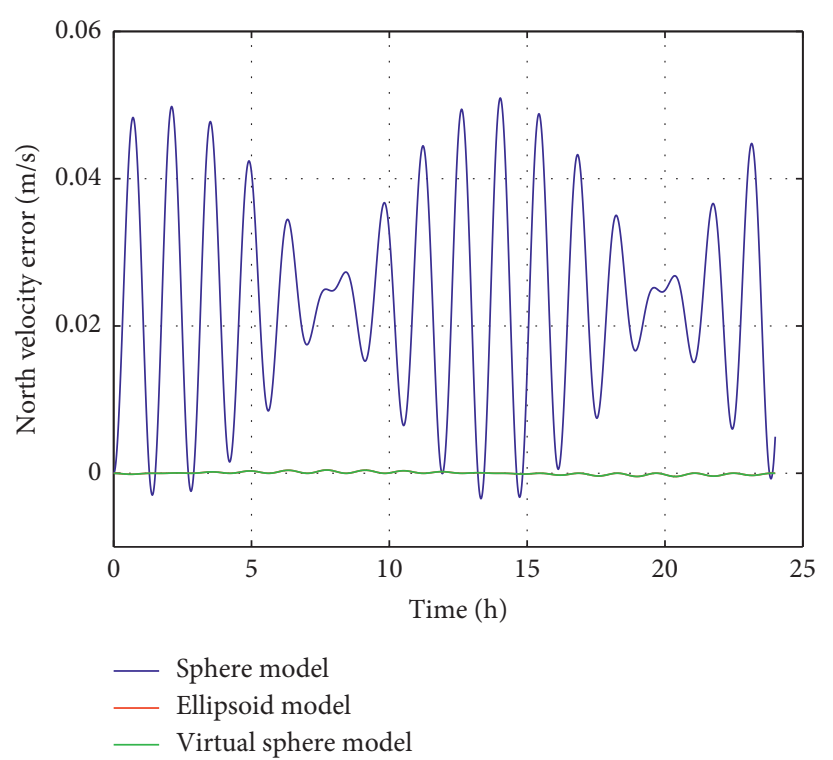

Figure 7: North velocity error.

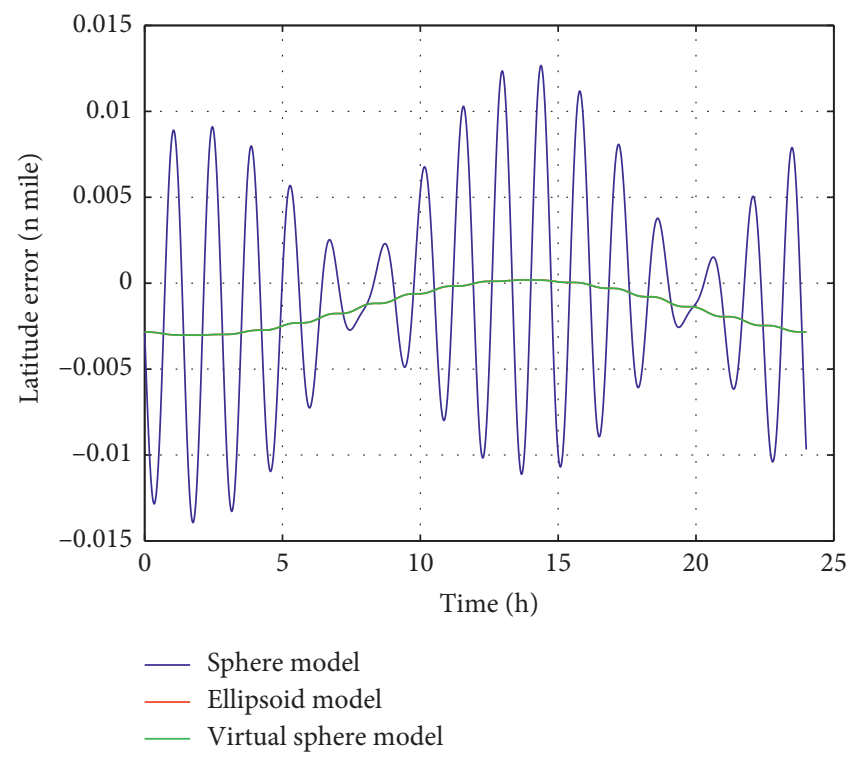

FiguRE 8: Latitude error.

navigation errors. The former two model methods are the traditional model methods and the third model method is the one proposed in this paper. Next, the performance of the three integrated navigation methods for AUV is compared by simulation.

Simulation conditions: the initial position of AUV: $\left(85^{\circ} \mathrm{N}, 118^{\circ} \mathrm{E}, 0 \mathrm{~m}\right)$; the north velocity is $6 \mathrm{~m} / \mathrm{s}$. The gyro drifts: $\varepsilon_{b}=\left(0.001^{\circ} / h\right)$, and the random drift of gyro: $\varepsilon_{w}=\left(0.001^{\circ} / \sqrt{h}\right)$; the accelerometer biases: $\nabla_{b}=100 \mathrm{ug}$, 


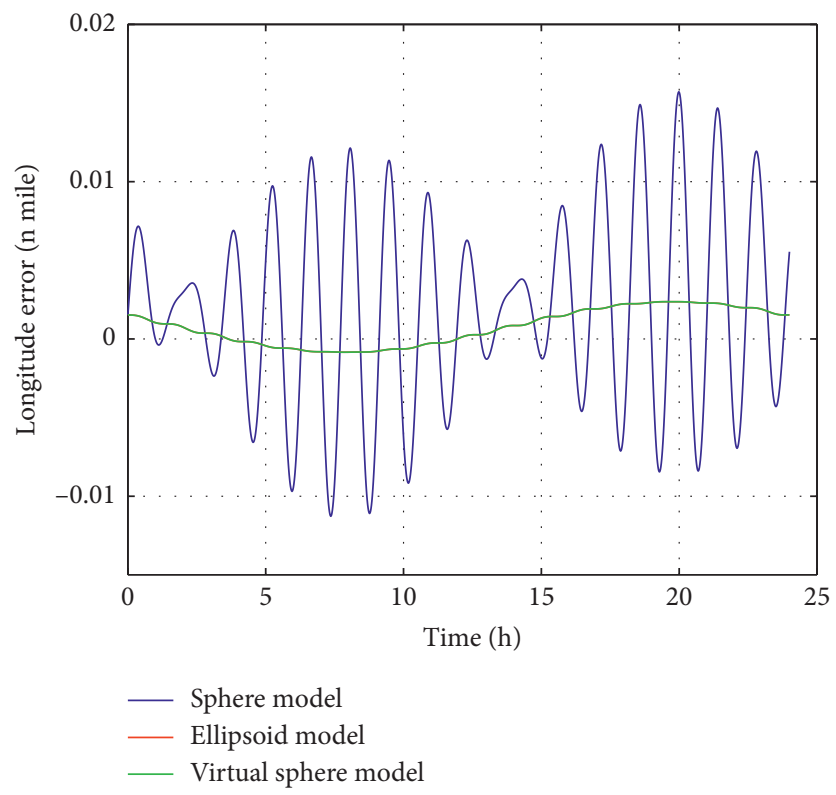

Figure 9: Longitude error.
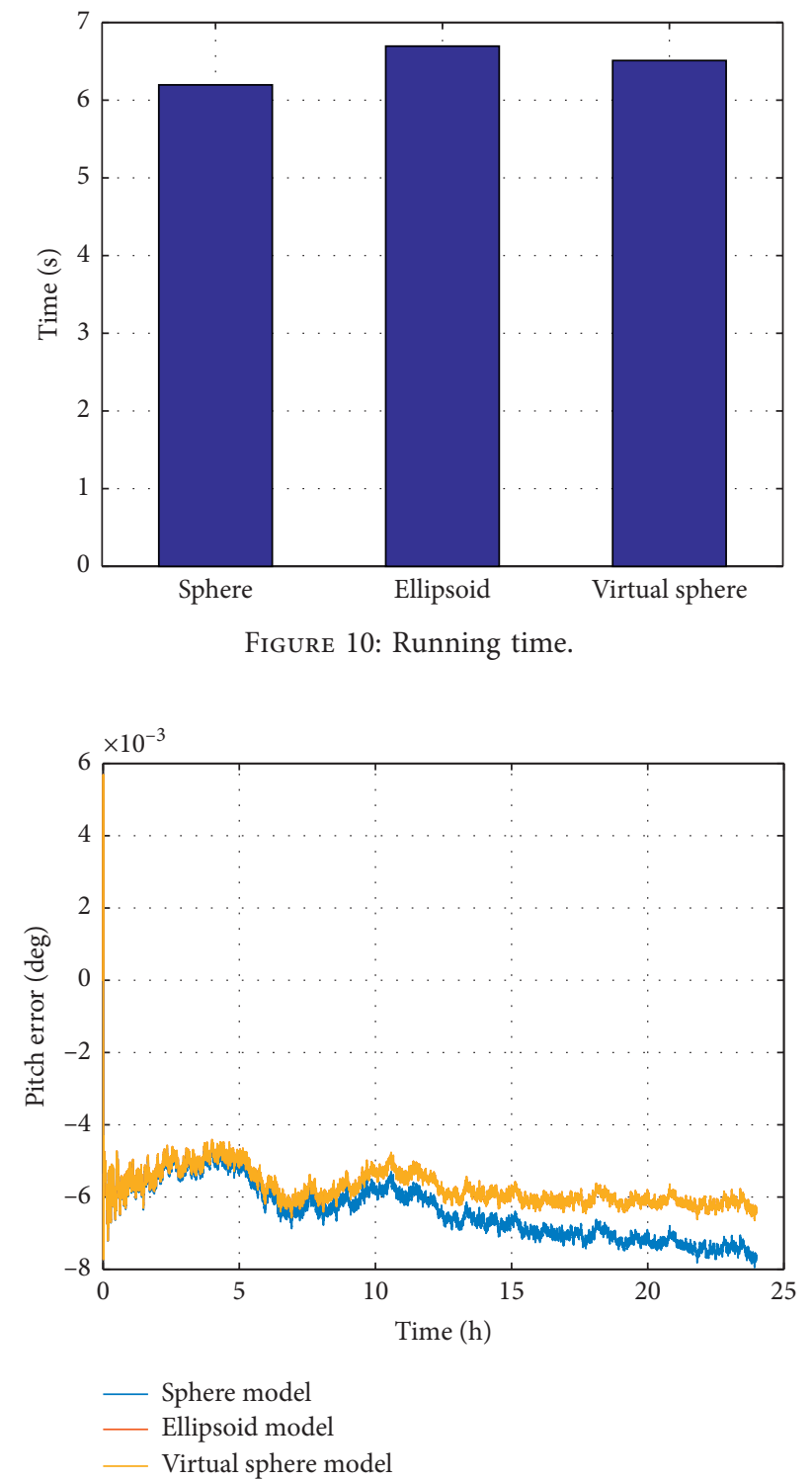

Figure 11: Pitch error. 


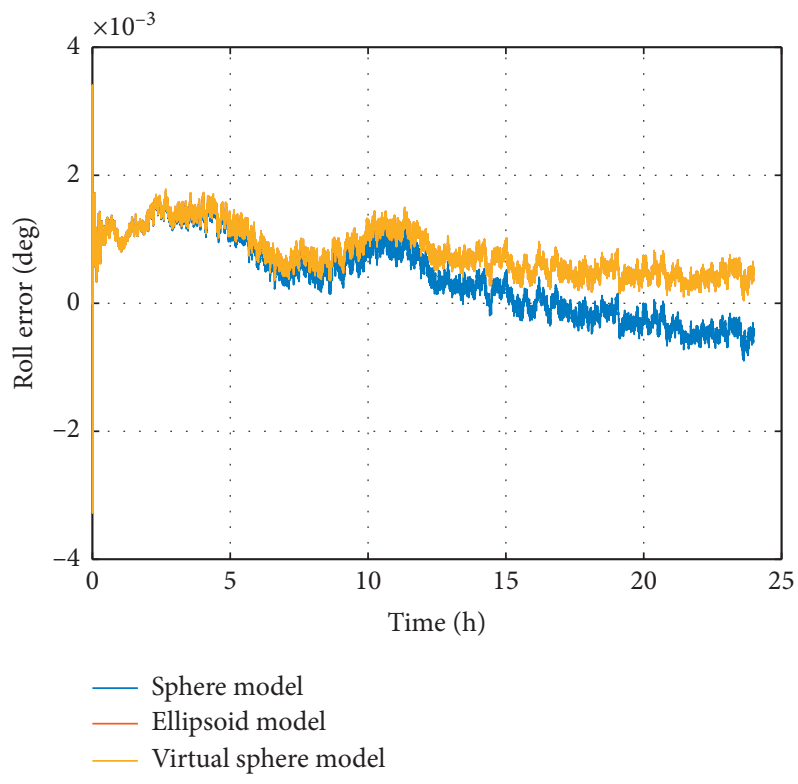

FIgURE 12: Roll error.

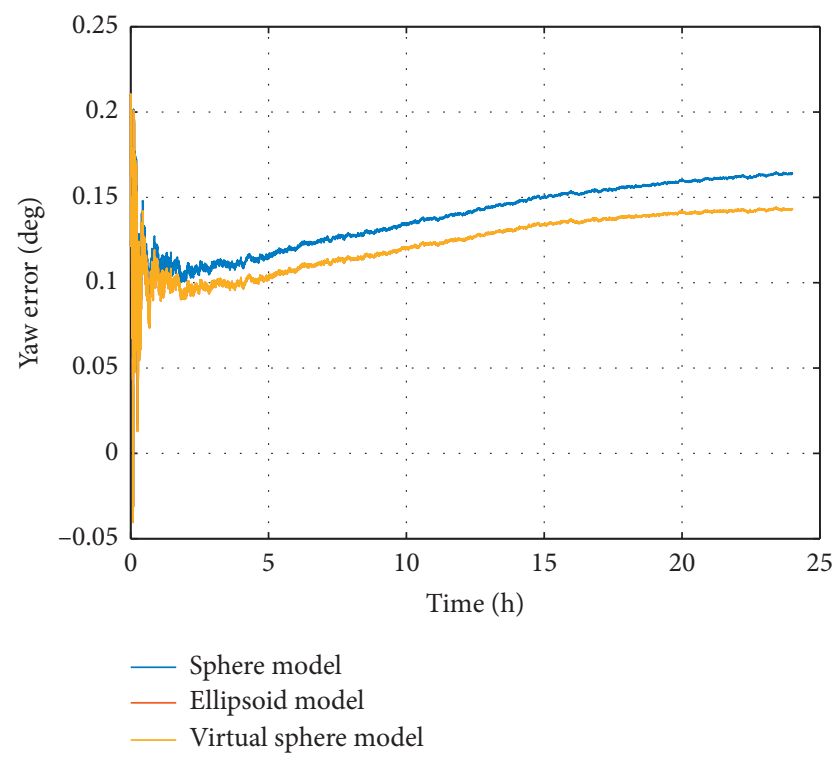

FIGURE 13: Yaw error.

and the random drift of accelerometer: $\nabla_{w}=(50 \mathrm{ug} / \sqrt{\mathrm{Hz}})$; the attitude error: $\left(30^{\prime \prime}, 30^{\prime \prime}, 20^{\prime}\right)$, the position error: $(1 \mathrm{~m}, 1 \mathrm{~m}, 3 \mathrm{~m})$, and the velocity error: $(0.1 \mathrm{~m} / \mathrm{s}, 0.1 \mathrm{~m} / \mathrm{s}, 0.1 \mathrm{~m} / \mathrm{s})$; the simulation time is $24 \mathrm{~h}$ and the filtering period is $1 \mathrm{~s}$. The simulation results are shown in Figures 11-17.

Simulation results demonstrate that the transverse SINS/ DVL integrated navigation based on the three Earth model methods can improve the navigation accuracy and suppress

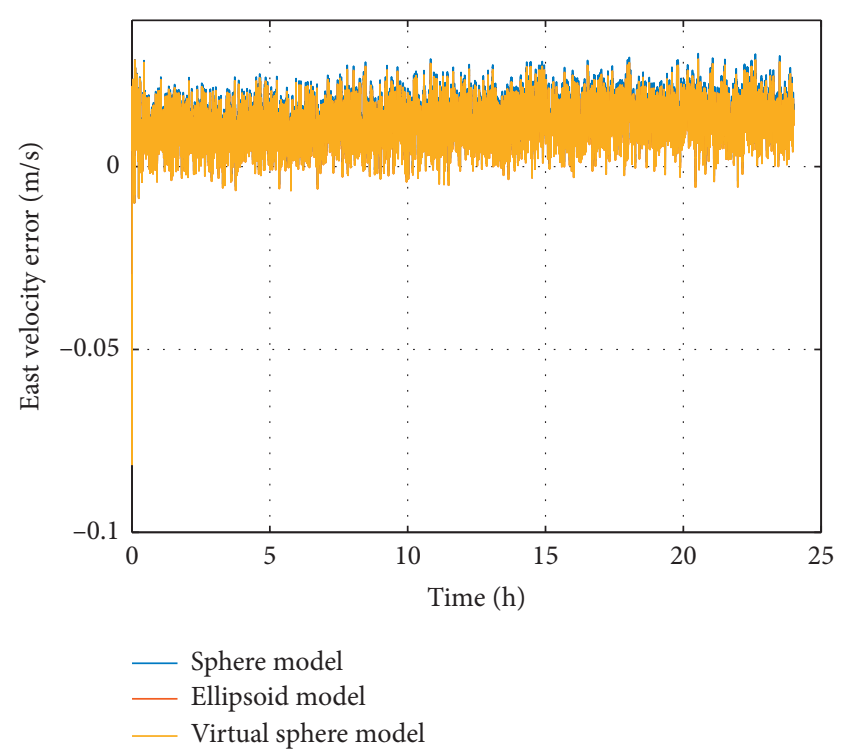

FIGURE 14: East velocity error.

the oscillation errors. As shown in Figures 11-17, the attitude errors of the integrated navigation algorithm based on the proposed virtual sphere model and the traditional sphere model are less than $0.01^{\circ}$, the yaw errors are less than $0.2^{\circ}$, the velocity errors are less than $0.04 \mathrm{~m} / \mathrm{s}$, and the position errors are less than $1000 \mathrm{~m}$ and $2500 \mathrm{~m}$, respectively. Compared with the traditional sphere model method, the proposed method has higher navigation accuracy and has almost similar accuracy with the traditional ellipsoid model 


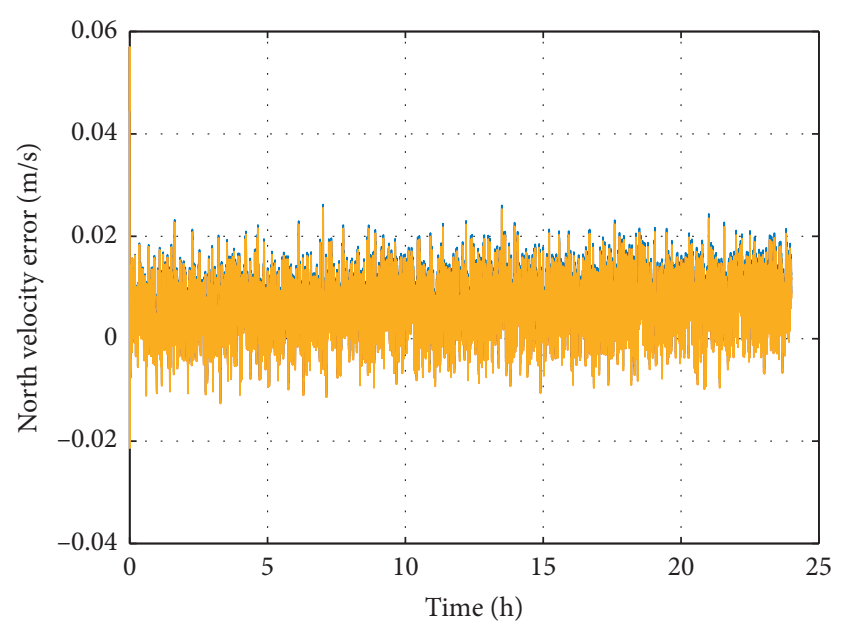

— Sphere model

— Ellipsoid model

_ Virtual sphere model

FIGURE 15: North velocity error.

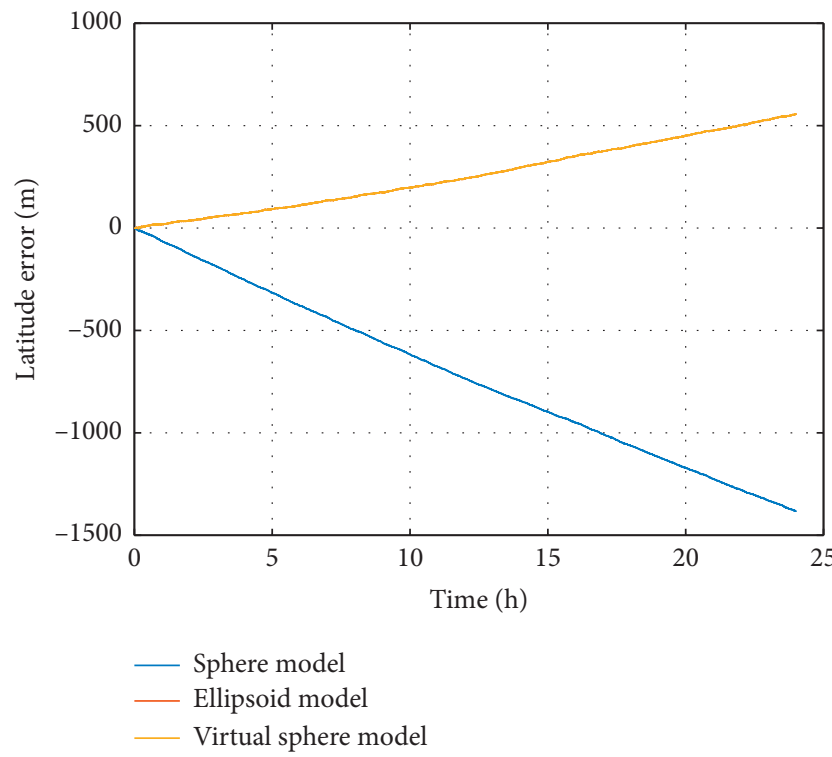

FIgURe 16: Latitude error.

method. Meanwhile, the virtual sphere model can be used to simplify the transverse transformation of the ellipsoid model. Therefore, the proposed transverse SINS/DVL integrated navigation system with the virtual sphere model has better performance than the traditional model methods. As shown in Figures 16 and 17, the level position error is divergent with time. For superlong-time underwater navigation, other auxiliary methods are needed to correct the position error of navigation.

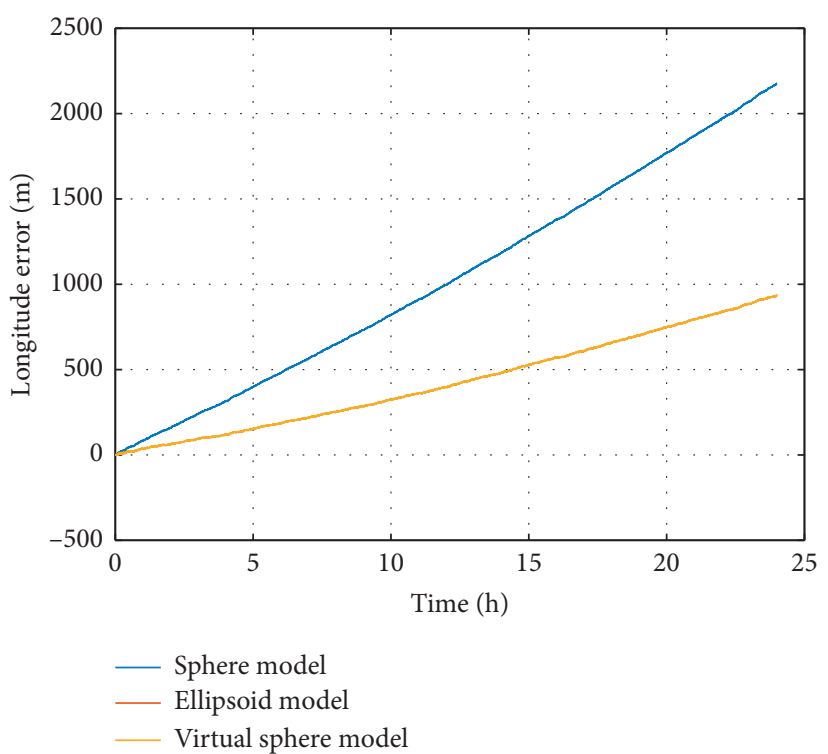

Figure 17: Longitude error.

\section{Conclusion}

The principle error introduced by the simplified Earth model is intolerable for high-precision navigation. To reduce the principle errors of the traditional sphere model, a new model is proposed, which is establishing a virtual sphere model with the radius of the prime vertical at the carrier position. In addition, the complex transverse transformation and derivation of the radius of the ellipsoid model is avoided by the proposed model. Compared with the transverse SINS based on the traditional sphere model, the accuracy of the transverse SINS based on the proposed virtual model is improved and has similar accuracy to that of the ellipsoid model. The proposed model in transverse SINS can not only suppress the oscillation errors but also reduce the constant velocity error. In order to further verify the effectiveness of the virtual sphere model, a method of transverse SINS/DVL integrated navigation system based on the virtual sphere model is proposed for AUV in the polar region. The simulation results show that the navigation accuracy of the integrated navigation method based on the virtual sphere model is better than that of the traditional sphere model method and coincides with the accuracy of the traditional ellipsoid model method. Finally, results indicate that the proposed method can satisfy the navigation accuracy of AUV in the polar region.

\section{Data Availability}

The data underlying the findings of the study are available from the corresponding author upon request. 


\section{Conflicts of Interest}

The authors declare that they do not have any conflicts of interest.

\section{Acknowledgments}

This work was supported in part by the National Natural Science Foundation of China under Grants 61803278 and in part by the Foundation of Key Laboratory of Micro-Inertial Instrument and Advanced Navigation Technology, Ministry of Education, China (SEU-MIAN-201802).

\section{References}

[1] R. Mcewen, H. Thomas, D. Weber, and F. Psota, "Performance of an AUV navigation system at arctic latitudes," IEEE Journal of Oceanic Engineering, vol. 30, no. 2, pp. 443-454, 2005.

[2] L. Stutters, H. Honghai Liu, C. Tiltman, and D. J. Brown, "Navigation technologies for autonomous underwater vehicles," IEEE Transactions on Systems, Man, and Cybernetics, Part C (Applications and Reviews), vol. 38, no. 4, pp. 581-589, 2008.

[3] L. Paull, S. Saeedi, M. Seto, and H. Li, "AUV navigation and localization: a review," IEEE Journal of Oceanic Engineering, vol. 39, no. 1, pp. 131-149, Jan. 2014.

[4] C. Broxmeyer, Inertial Navigation Systems, McGraw-Hill Book Company, New York, NY, USA, 1964.

[5] Q. LI, F. Sun, Y.-Y. Ben et al., "Transversal strapdown INS and damping design in polar region," Systems Engineering and Electronics, vol. 36, no. 12, pp. 2496-2503, 2014.

[6] Y.-Y. Qin, Inertial Navigation, Science Press, Beijing, China, 2nd edition, 2014.

[7] X.-S. Xu and M. Dou, "Inertial navigation algorithm in polar regions based on transverse geographic coordinate system," Huazhong University of Science and Technology (Natural Science Edition), vol. 12, pp. 116-121, 2014.

[8] Y.-Q. Yao, X.-S. Xu, Y. Li, Y.-T. Liu, J. Sun, and J.-W. Tong, "Transverse navigation under the ellipsoidal earth model and its performance in both polar and non-polar areas," Journal of Navigation, vol. 69, no. 2, pp. 335-352, 2016.

[9] Y.-S. Hao, A.-J. Zhou, and H.-B. Wang, "Analysis of the influence of the earth model of the transverse coordinate polar inertial navigation," Ship Electronic Engineering, vol. 37, no. 7, pp. 37-40, 2017.

[10] Q. Li, Y. Ben, F. Yu, and J. Tan, "Transversal strapdown INS based on reference ellipsoid for vehicle in the polar region," IEEE Transactions on Vehicular Technology, vol. 65, no. 9, pp. 7791-7795, 2016.

[11] H.-B. Wang, H.-W. Zhang, P.-P. Zhang et al., "Inertial navigation algorithm for polar region based on transverse terrestrial coordinate," Journal of Chinese Inertial Technology, vol. 24, no. 6, pp. 716-722, 2016.

[12] W.-C. Liu, H.-W. Bian, R.-Y. Wang et al., "Navigation performance of SINS transverse coordinate method," Geomatics and Information Science of Wuhan University, vol. 40, no. 11, pp. 1520-1525, 2015.

[13] X.-X. Lin, H.-W. Bian, H. Ma et al., "Applicability analysis of the approximate model of the earth with the arrangement of INS in polar region," Acta Geodaetica et Cartographica Sinica, vol. 48, no. 3, pp. 303-312, 2019.
[14] F.-J. Tan, L.-B. Chang, T. Lin et al., "Transverse polar navigation method based on virtual sphere model," Journal of Chinese Inertial Technology, vol. 26, no. 5, 2018.

[15] Z. Yan, Transversal Navigation Algorithm for Unmanned Underwater Vehicles in the Polar Region: Technical Committee on Control Theory, Chinese Association of Automation, Beijing, China, 2018.

[16] X. Liu, X. Xu, Y. Liu, and L. Wang, "A method for SINS alignment with large initial misalignment angles based on kalman filter with parameters resetting," Mathematical Problems in Engineering, vol. 2014, no. 11, Article ID 346291, 10 pages, 2014.

[17] F.-B. Zhang, P. Ma, and Z.-H. Wang, "SINS/DVL integrated navigation algorithm based on transversal coordinate frame in polar region," Acta Armamentarii, vol. 37, no. 7, pp. 12291235, 2016. 